\title{
Genotypic susceptibility to tipranavir (TPV) and darunavir (DRV) in a cohort of treatment-experienced patients (TEP) J Baxter*1, L Bhatti², J Scherer ${ }^{3}, \mathrm{M} \mathrm{McDonough}^{3}$ and P Piliero 3
}

\author{
Address: ${ }^{1}$ Cooper University Hospital/UMDNJ-Robert Wood Johnson Medical School, Camden, NJ, USA, ${ }^{2}$ AIDS Healthcare Foundation, Los \\ Angeles, CA, USA and ${ }^{3}$ Boehringer Ingelheim Pharmaceuticals Inc, Ridgefield, CT, USA \\ * Corresponding author
}

from Ninth International Congress on Drug Therapy in HIV Infection

Glasgow, UK. 9-13 November 2008

Published: 10 November 2008

Journal of the International AIDS Society 2008, I I (SuppI I):PI 92 doi:I0.I I86/I758-2652-I I-SI-PI 92

This abstract is available from: http://www.jiasociety.org/content/II/SI/PI 92

(c) 2008 Baxter et al; licensee BioMed Central Ltd.

\section{Purpose of the study}

TPV and DRV are ritonavir-boosted HIV protease inhibitors (PI) indicated for use in TEPs with PI resistance. Both agents have shown superior virologic and immunologic responses in TEPs compared with first-generation PIs. In the Utilization of HIV Drug Resistance in Treatment-Experienced Patients (UTILIZE) study, we assessed the presence of resistance to tipranavir, darunavir, and other antiretroviral agents among HIV-1 isolates in treatmentexperienced patients.

\section{Methods}

UTILIZE was an observational study at 40 US sites that examined clinician use of HIV drug-resistance testing in TEPs failing a PI-based regimen. Patients were randomized to have either a genotype (GT; Monogram GeneSeq) or combined phenotype-genotype test (PGT; Monogram Phenosense GT) to assist with treatment decision-making. For this analysis, only genotypic resistance data were evaluated to assess PI cross-resistance.

\section{Summary of results}

246 patients enrolled and 236 had resistance testing, of whom 139 (59\%) had evidence of HIV-1 resistance to at least one PI. Median HIV-RNA and CD4 count were 30,538 copies/ $\mathrm{mL}$ and 197 cells $/ \mathrm{mm}^{3}$, with no significant differences between GT and PGT groups. Of the 139 patients with evidence of PI resistance, $28 \%$ of isolates were resistant to all PIs; $58 \%$ and 55\% remained sensitive to TPV and DRV, respectively. In contrast, isolate susceptibility to indinavir, lopinavir, atazanavir, saquinavir, or nelfinavir was $21.6 \%, 20.1 \%, 19.4 \%, 16.5 \%$, and $7.9 \%$, respectively. TPV-DRV discordance rates were similar: $15.1 \%$ TPV sensitive/DRV resistant and $12.2 \%$ TPV resistant/DRV sensitive. 50\% (7/14) of isolates from TEPs failing TPV remained sensitive to TPV, while 3\% (1/29) of isolates from TEPs failing DRV remained sensitive to DRV.

\section{Conclusion}

In this cohort of TEPs failing a PI-based regimen, 59\% of HIV-1 isolates demonstrated PI resistance. Of these isolates, over $50 \%$ remained sensitive to either TPV or DRV, with $27.3 \%$ sensitive to only one of the two drugs. In PIexperienced patients, TPV and DRV remain the most likely available PIs to use in constructing a new regimen. 